ISSN 0103-9954

\title{
ANÁLISE DA REGENERAÇÃO NATURAL DE ESPÉCIES FLORESTAIS EM MATAS CILIARES DE ACORDO COM A DISTÂNCIA DA MARGEM DO LAGO
}

\section{ANALYSIS OF THE NATURAL REGENERATION OF FOREST SPECIES IN CILIARY WOODS BASED ON THE DISTANCE OF THE LAKE MARGINS}

\author{
José Carlos de Campos $^{1}$ Paulo Roberto Corrêa Landgraf ${ }^{1}$
}

\begin{abstract}
RESUMO
O presente trabalho teve como objetivo analisar a ocorrência da regeneração natural de espécies florestais nativas em mata ciliar em diferentes distâncias da margem do lago de Furnas. Considerou-se duas amostras, sendo uma com as parcelas localizadas a um metro da margem do lago e a outra distante 36 metros. As plantas foram identificadas, medindo-se os valores de diâmetro do colo, altura total e densidade. No estrato próximo ao lago, encontrou-se uma densidade de 3770 plantas/ha distribuídas em 34 espécies e 17 famílias, com altura média de 1,15 metros e diâmetro médio do colo de $0,97 \mathrm{~cm}$. Já na amostra distante do lago encontrou-se uma densidade de 4510 plantas/ha distribuídas em 29 espécies e 17 famílias, com altura média das plantas de 1,43 metros e diâmetro médio do colo de 1,25 centímetros. Na amostra distante do lago, encontrou-se menor número de espécies, porém com maior densidade e maiores valores de diâmetro do colo e altura.
\end{abstract}

Palavras-chave: Mata ciliar, regeneração natural.

\begin{abstract}
The purpose of this paper was to analyse the occurrence of natural regeneration of native forest species in ciliary wood at different distances from the margins of the Furnas lake.Two samples were considered: one with parcels located one meter from the margin of the lake, and the other at 36 meters. The plants were identified, measuring the values of the diameter of the lowest base, total height, and density. In the sample next to the lake, 0 a density of 3,770 plants per hectare was found, distributed in 34 species and 17 families, with the average height of 1,15 meters and an average diameter of the lowest base of 0,97 centimeters. In the sample distant from the lake, a density of 4,510 plants per hectare was found, distributed into 29 species and 17 families, with the average height of 1,43 meters and an average diameter of 1,25 centimeters for the lowest base. In the distant sample, there was a smaller number of species, but high ter density and higher values for the height and diameter of the lowest base.
\end{abstract}

Key words: Ciliary wood, natural regeneration.

\section{INTRODUÇÃO}

A exploração florestal no sul do Estado de Minas Gerais sempre foi intensa, principalmente

1. Engenheiro Florestal, MSc. Professor do Instituto de Ciências Agrárias, Universidade de Alfenas, Caixa Postal 23, CEP 37130-000, Alfenas (MG). landgraf@unifenas.br 
na região mais central. Na área mais próxima ao Reservatório de Furnas, a cobertura florestal nativa não ultrapassa a 6\% e é composta por fragmentos florestais, na sua maioria, inferiores a 30 ha (CAMPOS \& LANDGRAF, 1990). A devastação florestal provocou muitos danos à flora, à fauna silvestre, além de prejuízos irreparáveis aos recursos hídricos e edáficos.

Muitas espécies florestais se encontram em vias de extinção principalmente em consequência do seu valor econômico e do sistema seletivo de exploração. Assim sendo, tornam-se necessários levantamentos e estudos específicos sobre o comportamento dessas matas nativas, principalmente aquelas situadas à margem dos cursos d'água. A presença de matas nativas, principalmente nas encostas íngremes, topo de morros e ao longo de rios, córregos e represas serve como obstáculo ao livre escorrimento da água das enxurradas, reduzindo sua velocidade e possibilitando sua infiltração no solo para absorção pelas plantas e para alimentação dos aquíferos subterrâneos. Conseqüentemente, contribui de maneira decisiva para evitar o assoreamento do leito de córregos, rios, estuários, lagoas e várzea.

A biodiversidade das florestas tropicais inclui não somente o número de espécies, mas também a diversidade genética e de habitats. A manutenção do número de populações e sua variação genética são os objetos de estudos atuais em conservação. Investigações recentes deram grande importância as respostas ecológicas de populações sobre pressão de fragmentação e também ao conhecimento e incorporação desses dados em modelos de conservação e reconhecimento aplicados em áreas degradadas (BARBOSA, 1997).

GANDOLFI (1991) relata que os estudos das florestas tropicais têm crescido nas últimas décadas, não apenas com relação à descrição da composição florística e estrutura fitossociológica, mas também buscando entender a dinâmica desses ecossistemas. A regeneração das espécies vegetais é um processo natural em que cada espécie desenvolve características próprias, em perfeita sintonia com as condições ambientais. A dinâmica natural permite a perpetuação de todas as espécies vegetais através dos tempos. A extinção de espécies ocorreu naturalmente em virtude de alterações graduais do ambiente durante milhares de anos, (SEITZ, 1994).

Segundo INOUE (1979), a regeneração natural ocorre no sistema de alto fuste, iniciando-se pela maturação e germinação da semente, atingindo o estágio de crescimento que suporta a concorrência com as outras espécies. A garantia da permanência de uma determinada espécie em uma floresta é função direta do número de indivíduos e de sua distribuição nas classes de diâmetro. Desta forma, uma densidade populacional baixa significa que existe uma possibilidade maior dessa espécie ser substituída por outra no desenvolvimento da floresta, por razões naturais ou em razão das perturbações ocorridas na área.

PINHEIRO et al. (1989) relatam que o conhecimento e identificação de mudas não é simples, principalmente porque as características morfológicas externas de uma planta jovem podem ser diferentes daquelas observadas nos indivíduos adultos. Para SEITZ (1994), a regeneração natural tem recuperado grandes áreas de vegetação degradada durante os séculos passados, tanto em função da ação antrópica quanto em consequência de cataclismas naturais. No processo de regeneração natural, as características das espécies, principalmente quanto à dispersão das sementes e sua posição dentro das fases, são de principal importância, definindo o sucesso ou insucesso de um programa de recuperação de áreas degradadas.

Ciência Florestal, v. 11, n. 2, 2001 
FINGER (1977) descreveu e identificou mudas, em regeneração natural de 50 espécies florestais nativas na região de Viçosa - MG, estabelecendo correlação de caracteres morfológicas com plantas adultas.

O presente trabalho teve como objetivo efetuar o levantamento e a análise das espécies florestais nativas em regeneração natural em uma mata nativa ciliar no reservatório de Furnas, em duas diferentes distâncias com relação à margem do lago.

\section{MATERIAL E MÉTODOS}

\section{Caracterização do local}

A área pesquisada localiza-se entre os paralelos $21^{\circ} 00^{\prime}$ e $21^{\circ} 30^{\prime}$ de latitude sul e meridianos $45^{\circ} 30^{\prime}$ e $46^{\circ} 00^{\prime}$ W.Gr., na região de transição entre a mata atlântica e o cerrado, na margem do lago de Furnas em Minas Gerais. A altitude da região varia de $769 \mathrm{~m}$ a $1000 \mathrm{~m}$, com relevo de ondulado a montanhoso. O clima é subtropical moderado úmido com precipitação média anual de 1400 a $1700 \mathrm{~mm}$, tendo como vegetação predominante a floresta perenifólia e subperenifólia com ocorrência de cerrado (GOLFARI, 1980). De acordo com a classificação regional vegetal feita pelo IBGE (1993), citado por ALVARENGA (1998), a cobertura florestal enquadra-se na floresta estacional semidecidual.

\section{Estabelecimento das amostra e medição em campo}

Para a realização da pesquisa, selecionou-se uma mata nativa à margem do lago com características típicas da região, estabelecendo-se dois estratos nas proximidades do lago, tendo cada um 10 parcelas de $5 \times 10$ metros. As parcelas do primeiro estrato (A) foram demarcadas a um metro da margem do reservatório com o maior comprimento paralelo à margem e eqüidistantes a pelo menos 100 metros. As parcelas do segundo estrato (B) foram localizadas a 36 metros da margem do lago e paralelas às do primeiro estrato. Com a demarcação das parcelas, essas foram qualificadas em 1A, 1B, 2A, 2B e, assim, sucessivamente onde o número corresponde a ordem de parcelas e a letra da a posição do estrato em relação à margem do reservatório, conforme Figura 1.

De acordo com a descrição morfológica do perfil no campo e com resultados de análises laboratoriais das amostras coletadas, o solo da área pesquisada foi classificado em Glei Pouco Húmico Eutrófico A Chernozênico, textura argilosa. A umidade do solo medida na época da avaliação em campo (agosto) foi de $9,12 \%$ no estrato A e $8,39 \%$ no estrato B. A declividade média do terreno nas áreas pesquisadas foi de $43,6 \%$ no estrato A e $13,8 \%$ no estrato B.

Para o desenvolvimento da pesquisa e facilitar a tomada das informações em campo, definiuse, como plantas na condição de regeneração natural todas as espécies arbóreas nativas com DAP (diâmetro a altura do peito) inferior a $5,0 \mathrm{~cm}$, independente do valor da altura total. Em cada unidade de amostra, as árvores qualificadas foram identificadas com o seu nome comum, medindo- se os valores de altura total com uma régua graduada e diâmetro de base com uma suta, anotando-se em fichas específicas todas as informações observadas em campo. Classificaram-se as plantas da 
regeneração natural com o seu nome científico e família, calculando-se os valores médios de diâmetro da base e altura total bem como a densidade por espécie e total para cada estrato.

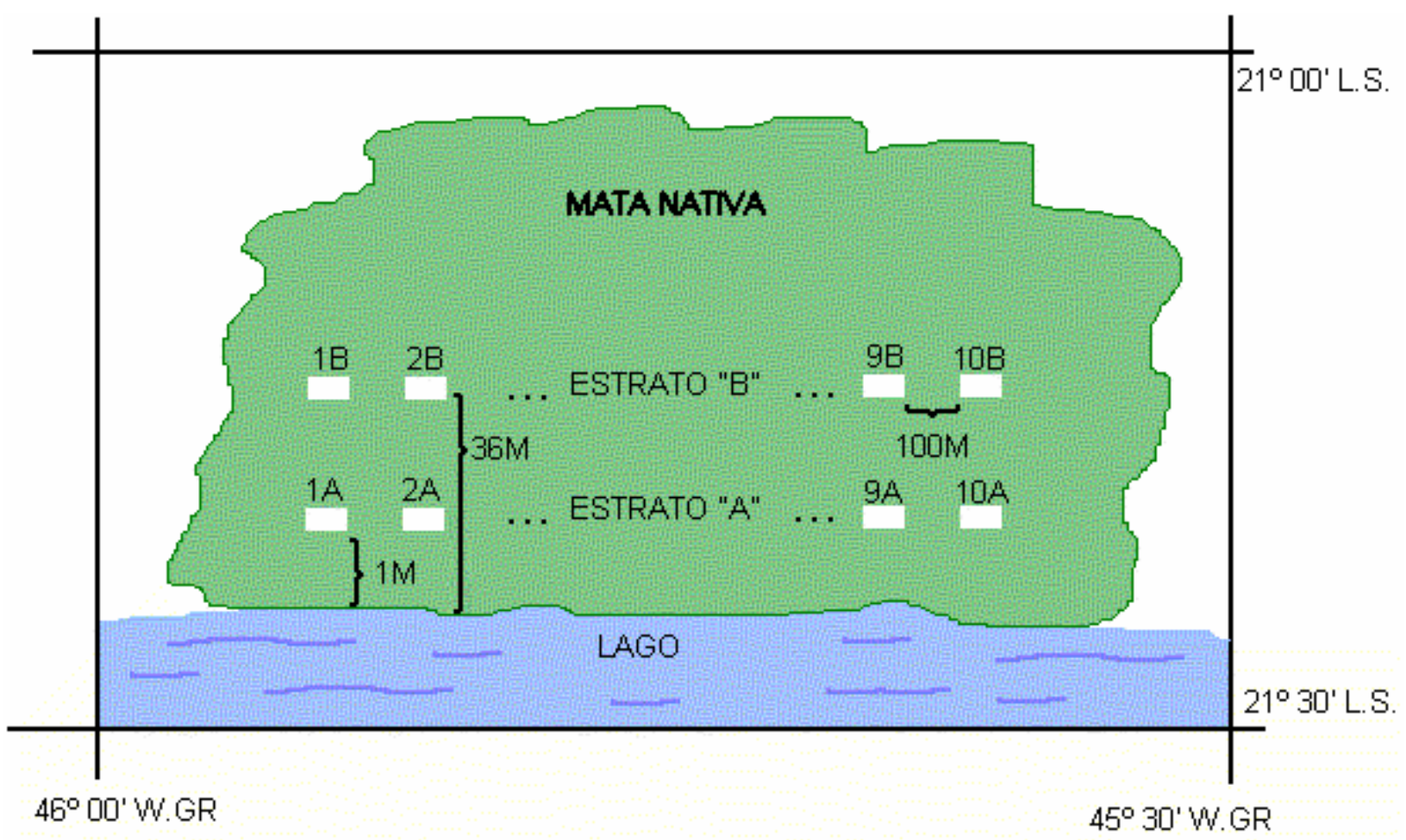

FIGURA 1: Localização da área da pesquisa e distribuição das amostras.

\section{RESULTADOS E DISCUSSÃO}

$\mathrm{Na}$ Tabela 1, apresentam-se as espécies florestais com regeneração natural classificadas por estrato, enquanto que na Tabela 2, estão os valores médios de densidade, diâmetro da base e altura média para cada espécie por estrato na floresta marginal do lago de Furnas.

TABELA 1: Espécies florestais com regeneração natural em uma floresta marginal do lago de Furnas. Alfenas - MG, 1998.

\begin{tabular}{lllc}
\hline Nome comum & Nome científico & Família & Estrato \\
\hline Angico & Parapiptadenia rigida (Benth.) Brenan & Mimosaceae & A \\
Armescla & Protium heptaphyllum (Aubl.) March & Burseraceae & A \\
Cafezinho & Rhamnidium elaecarpus Reiss & Rhamnaceae & A \\
Canela amarela & Nectandra lanceolata Ness. Et Mart. Ex Nees & Lauraceae & A \\
Canela de urubu & Ocotea megapotamica (Spreng.) Mez & Lauraceae & A \\
Espinheira santa & Pachystroma longifolium (Ness) I.M. Johns & Euphorbiaceae & A \\
\hline
\end{tabular}

Continua ...

Ciência Florestal, v. 11, n. 2, 2001 
TABELA 1: Continuação ...

\begin{tabular}{|c|c|c|c|}
\hline Nome comum & Nome científico & Família & Estrato \\
\hline Folha miuda & Pterocarpus violaceus Vog. & Fabaceae & $\mathrm{A}$ \\
\hline Jacarandá & Dalbergia miscolobium Benth & Fabaceae & A \\
\hline Lixa & Aloysia virgata (Ruiz et Pav.) ${ }^{a}$ L. Juss. & Verbenaceae & A \\
\hline Marmelinho & Austroplenckia populnea (Reiss.) Lund. & Celastraceae & A \\
\hline Morototó & Bauhinia sp & Caesalpiniaceae & A \\
\hline Pinho do brejo & Talauma ovata St. Hil. & Magnoliaceae & A \\
\hline Pororoca & Rapanea ferruginea (Ruiz et Pav.) Mez & Myrsinaceae & A \\
\hline Veludo & Sclerolobium sp. & Caesalpiniaceae & B \\
\hline Açoita cavalo & Luehea divaricata Mart & Tiliaceae & B \\
\hline Canjica & Sweetia fruticosa Spreng. & Fabaceae & B \\
\hline Carne de vaca & Roupala brasiliensis Klots & Proteaceae & B \\
\hline Cedro & Cedrela fissilis Vell. & Meliaceae & B \\
\hline Rabo de bugio & Lonchocarpus muehlbergianus HassL & Fabaceae & B \\
\hline Peroba & Aspidosperma polyneuron M. Arg. & Apocynaceae & B \\
\hline $\begin{array}{l}\text { Laranjeira de } \\
\text { vaqueiro }\end{array}$ & Zizyphus sp. & Rhamnaceae & B \\
\hline Angazinho & Inga sp Hooker at Arnott & Mimosaceae & $\mathrm{A} / \mathrm{B}$ \\
\hline Camboatá & Guarea guidonia (L.) Sleumer & Meliaceae & $\mathrm{A} / \mathrm{B}$ \\
\hline Cambuí & Myrciaria tenella (DC.) Berg & Myrtaceae & $\mathrm{A} / \mathrm{B}$ \\
\hline Canela sassafraz & Ocotea odorifera (Vell.) Mez & Lauraceae & $\mathrm{A} / \mathrm{B}$ \\
\hline Canjerana & Cabralea canjerana (Vell.) Mart. & Meliaceae & $\mathrm{A} / \mathrm{B}$ \\
\hline Branquilho & $\begin{array}{l}\text { Sebastiania commersoniana (Baill.) Smith \& } \\
\text { Downs }\end{array}$ & Euphorbiaceae & $\mathrm{A} / \mathrm{B}$ \\
\hline Catiguá Vermelho & Trichilia claussenii C. DC. & Meliaceae & $\mathrm{A} / \mathrm{B}$ \\
\hline Erva e lagarto & Casearia silvestris $\mathrm{Sw}$. & Flacourtiaceae & $\mathrm{A} / \mathrm{B}$ \\
\hline Espeto & Casearia gossypiosperma Briquet. & Flacourtiaceae & $\mathrm{A} / \mathrm{B}$ \\
\hline Pau viola & Cytharexyllum myrianthum Cham. & Verbenaceae & $\mathrm{A} / \mathrm{B}$ \\
\hline Ingá & Inga uruguensis Hooker at Arnott & Mimosaceae & $\mathrm{A} / \mathrm{B}$ \\
\hline Jambo & Eugenia $s p$ & Myrtaceae & $\mathrm{A} / \mathrm{B}$ \\
\hline Macoqueiro & Aspidosperma sp & Apocynaceae & $\mathrm{A} / \mathrm{B}$ \\
\hline Mamoninho & Mabea fistulifera Mart. & Euphorbiaceae & $\mathrm{A} / \mathrm{B}$ \\
\hline Monjoleiro & Acacia polyphylla DC. & Mimosaceae & $\mathrm{A} / \mathrm{B}$ \\
\hline Óleo copaíba & Copaifera langsdorffii Desf. & Caesalpiniaceae & $\mathrm{A} / \mathrm{B}$ \\
\hline Peito de pomba & Tapirira guianensis Aubl. & Anacardiaceae & $\mathrm{A} / \mathrm{B}$ \\
\hline Pereira & Platycyamus regnelli Vog. & Fabaceae & $\mathrm{A} / \mathrm{B}$ \\
\hline Pessegueiro & Prunus sellowii Koehne & Rosaceae & $\mathrm{A} / \mathrm{B}$ \\
\hline Roxinho & Peltogyne angustiflora Ducke & Caesalpiniaceae & $\mathrm{A} / \mathrm{B}$ \\
\hline Sangra d água & Croton urucurana Baill. & Euphorbiaceae & $\mathrm{A} / \mathrm{B}$ \\
\hline
\end{tabular}

Considerando-se os resultados apresentados na Tabela 1, pode-se observar a diversidade de espécies florestais identificadas nos dois estratos. No estrato A, foram encontradas 34 espécies agrupadas em 17 famílias, enquanto que no estrato B identificaram-se 29 espécies pertencentes a 17 famílias. Deste total, 21 espécies aparecem tanto no estrato A como no estrato B, enquanto 13 espécies só foram encontradas no estrato A, e 8 espécies somente no estrato B. 
TABELA 2: Classificação dos valores de densidade, diâmetro da base e altura média das espécies por estrato.

\begin{tabular}{|c|c|c|c|c|c|c|}
\hline \multirow[t]{2}{*}{ Nome científico } & \multicolumn{3}{|c|}{$\begin{array}{l}\text { Estrato } \\
\text { "A" }\end{array}$} & \multicolumn{3}{|c|}{$\begin{array}{l}\text { Estrato } \\
\text { "B" }\end{array}$} \\
\hline & DENS/ha & DB (cm) & Alt. (m) & DENS/ha & $\mathrm{DB}(\mathrm{cm})$ & Alt. (m) \\
\hline Luehea divaricata Mart & - & - & 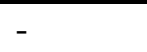 & 20 & 0,10 & 0,10 \\
\hline Inga sp Hooker at Arnott & 40 & 0,25 & 0,23 & 300 & 1,19 & 1,11 \\
\hline Parapiptadenia rigida (Benth.) Brenan & 20 & 0,16 & 0,28 & - & - & - \\
\hline Protium heptaphyllum (Aubl.) March & 40 & 0,90 & 1,07 & - & - & - \\
\hline Rhamnidium elaecarpus Reiss & 40 & 0,73 & 0,55 & - & - & - \\
\hline Guarea guidonia (L.) Sleumer & 960 & 2,33 & 3,06 & 820 & 2,87 & 3,76 \\
\hline Myrciaria tenella (DC.) Berg & 140 & 0,85 & 0,85 & 140 & 1,12 & 1,38 \\
\hline Ocotea odorifera (Vell.) Mez & 190 & 2,65 & 4,33 & 320 & 2,92 & 3,84 \\
\hline $\begin{array}{l}\text { Nectandra lanceolata Ness. Et Mart. Ex } \\
\text { Nees }\end{array}$ & 20 & 0,42 & 0,55 & - & - & - \\
\hline Ocotea megapotamica (Spreng.) Mez & 20 & 0,14 & 0,20 & - & - & - \\
\hline Cabralea canjerana (Vell.) Mart & 30 & 2,48 & 2,63 & 340 & 2,87 & 2,59 \\
\hline Sweetia fruticosa Spreng. & - & - & - & 40 & 0,71 & 1,00 \\
\hline Sebastiania commersoniana (Baill.) & 20 & 0,21 & 0,16 & 20 & 0,60 & 0,65 \\
\hline Smith \& Downs & & & & & & \\
\hline Roupala brasiliensis Klots & - & - & - & 20 & 0,47 & 0,40 \\
\hline Trichilia claussenii C. DC. & 240 & 2,10 & 2,27 & 180 & 2,96 & 3,56 \\
\hline Cedrela fissilis Vell. & - & - & - & 40 & 0,45 & 0,30 \\
\hline Casearia silvestris $\mathrm{Sw}$. & 30 & 1,09 & 1,38 & 40 & 0,88 & 1,10 \\
\hline Casearia gossypiosperma Briquet. & 100 & 1,22 & 1,28 & 40 & 0,58 & 0,93 \\
\hline $\begin{array}{l}\text { Pachystroma longifolium (Ness) I.M. } \\
\text { Johns }\end{array}$ & 160 & 2,13 & 1,99 & - & - & - \\
\hline Pterocarpus violaceus Vog. & 40 & 0,28 & 0,40 & - & - & - \\
\hline Inga uruguensis Hooker at Arnott & 40 & 0,27 & 0,45 & 40 & 0,72 & 1,10 \\
\hline Dalbergia miscolobium Benth & 20 & 0,61 & 0,41 & - & - & - \\
\hline Eugenia $s p$ & 340 & 2,34 & 2,90 & 300 & 1,84 & 2,02 \\
\hline Zizyphus sp. & - & - & - & 20 & 0,65 & 0,80 \\
\hline Aloysia virgata (Ruiz et Pav.) ${ }^{\mathrm{a}} \mathrm{L}$. Juss. & 20 & 0,14 & 0,20 & - & - & - \\
\hline Aspidosperma sp & 200 & 1,34 & 1,37 & 20 & 0,22 & 0,28 \\
\hline Mabea fistulifera Mart. & 40 & 0,86 & 0,66 & 240 & 2,39 & 2,50 \\
\hline Bauhinia sp & 20 & 0,75 & 0,80 & - & - & - \\
\hline Acacia polyphylla DC. & 30 & 0,83 & 1,66 & 160 & 1,62 & 1,89 \\
\hline Bauhinia sp & 20 & 0,31 & 0,40 & - & - & - \\
\hline Copaifera langsdorffii Desf. & 20 & 0,15 & 0,31 & 20 & 0,24 & 0,32 \\
\hline Cytharexyllum myrianthum Cham. & 20 & 1,69 & 2,01 & 30 & 2,68 & 3,12 \\
\hline Tapirira guianensis Aubl. & 30 & 2,02 & 2,39 & 460 & 2,90 & 3,06 \\
\hline Platycyamus regnelli Vog. & 480 & 0,83 & 1,07 & 380 & 0,48 & 0,63 \\
\hline Aspidosperma polyneuron M. Arg. & - & - & - & 60 & 0,11 & 0,18 \\
\hline Prunus sellowii Koehne & 20 & 0,31 & 0,35 & 40 & 1,59 & 2,12 \\
\hline
\end{tabular}

Continua ...

Ciência Florestal, v. 11, n. 2, 2001 
TABELA 2: Continuação ...

\begin{tabular}{|c|c|c|c|c|c|c|}
\hline \multirow[t]{2}{*}{ Nome científico } & \multicolumn{3}{|c|}{$\begin{array}{c}\text { Estrato } \\
\text { "A" }\end{array}$} & \multicolumn{3}{|c|}{$\begin{array}{l}\text { Estrato } \\
\text { "B" }\end{array}$} \\
\hline & DENS/ha & $\mathrm{DB}(\mathrm{cm})$ & Alt. (m) & DENS/ha & $\mathrm{DB}(\mathrm{cm})$ & Alt. (m) \\
\hline Talauma ovata St. Hil. & 20 & 0,22 & 0,31 & - & - & - \\
\hline Rapanea ferruginea (Ruiz et Pav.) Mez & 20 & 0,84 & 0,74 & - & - & - \\
\hline Lonchocarpus muehlbergianus HassL & - & - & - & 20 & 0,24 & 0,31 \\
\hline Peltogyne angustiflora Ducke & 320 & 0,97 & 1,11 & 360 & 1,90 & 1,86 \\
\hline Croton urucurana Baill. & 20 & 0,51 & 0,75 & 20 & 0,41 & 0,32 \\
\hline Sclerolobium $s p$ & - & - & - & 20 & 0,62 & 0,30 \\
\hline Total & 3770 & 32,93 & 39,12 & 4510 & 36,33 & 41,53 \\
\hline Média & 110,88 & 0,97 & 1,15 & 155,52 & 1,25 & 1,43 \\
\hline Número de espécies & 34 & - & - & 29 & - & - \\
\hline
\end{tabular}

Com relação à distribuição de espécies por estrato, verificou-se que espécies como açoita cavalo (Luehea divaricata), cangica (Sweetia fruticosa), carne de vaca (Roupala brasiliensis), cedro (Cedrela fissilis), laranjeira de vaqueiro (Zizyphus sp), pessegueiro (Prunus sellowii), rabo de bugio (Lonchocarpus muehlbergianus) e veludo (Sclerolobium $s p$ ) só ocorreram nas parcelas mais distantes do lago. Isso pode ser atribuído ao fato de que tais espécies não toleram solos úmidos. Algumas espécies só foram encontradas no estrato mais próximo ao lago, dentre elas se pode citar o angico (Parapiptadenia rigida), amescla (Protium heptaphyllum), cafezinho (Rhamnidium elaecarpus), canela amarela (Nectandra lanceolata), canela de urubu (Ocotea megapotamica), espinheira santa (Pachystroma longifolium), folha miúda (Pterocarpus violaceus), jacarandá (Dalbergia miscolobium), lixa (Aloysia virgata), marmelinho (Austroplenckia populnea), morototó (Bauhinia sp), pororoca (Rapanea ferruginia) e pinho do brejo (Talauma ovata). SPINA E LEITÃO FILHO (1995) detectaram a ocorrência de pinho do brejo em uma área com encharcamento permanente de solo. RAMOS et al. (1995) verificaram a presença de Talauma ovata entre as espécies dominantes em locais encharcados em uma mata de galeria em Brasília. Essas espécies apresentam comportamento de espécies que se desenvolvem nas proximidades de curso d'água, conforme OLIVEIRA FILHO (1995).

De acordo com os resultados apresentados no Tabela 2, nota-se uma média de 3.770 plantas por hectare no estrato A, com altura média das plantas de $1,15 \mathrm{~m}$ e diâmetro médio da base de $0,97 \mathrm{~cm}$. A espécie com maior densidade foi o camboatá (Guarea guidonia) com 960 plantas por hectare, seguido pela pereira (Platycyamus regnelli) com 480 plantas por hectare e o jambo (Eugenia sp.) com 340 plantas por hectare.

No estrato B, foram qualificadas em média 4.510 plantas por hectare, com altura média das plantas de $1,43 \mathrm{~m}$ e diâmetro da base de $1,25 \mathrm{~cm}$. A espécie com maior densidade foi o camboatá (Guarea guidonia) com 820 plantas por hectare, seguido pelo peito de pomba (Tapirira guianensis) com 460 plantas por hectare e pereira (Platycyamus regnelli) com 380 plantas por hectare.

Analisando-se os estratos A e B, pode-se observar que espécies de grande valor econômico foram encontradas, como o jacarandá (Dalbergia microlobium) apresentou densidade de 20 árvores por hectare, aparecendo apenas no estrato próximo à margem do lago. O cedro (Cedrela fissilis) só 
apareceu no estrato mais distante da margem do lago com densidade de 40 plantas por hectare. A peroba (Aspidosperma polyneuron) apareceu com maior densidade no B. O óleo copaíba (Copaifera langsdorfii) foi encontrado nos dois estratos com mesma densidade, ou seja, 20 plantas por hectare. Além disso, verificou-se que as espécies do estrato A apresentaram menor valor médio de diâmetro da base $0,97 \mathrm{~cm}$ e altura de $1,15 \mathrm{~m}$ quando comparado com estrato B que apresentou diâmetro médio da base de $1,25 \mathrm{~cm}$ e altura média $1,43 \mathrm{~m}$.

Algumas espécies foram encontradas com grande diferença de densidade entre os estratos; o ingazinho (Inga $\mathrm{sp}$ ) apresentou densidade média de 40 plantas/ha no estrato A e 300 plantas/ha no estrato B. A canjerana (Cabralea canjerana) com valores médios por hectare de 30 plantas no estrato A e 340 plantas no estrato B. Esse resultado, discorda de SALVADOR (1987), em que o autor caracterizou o Inga $s p$ como espécie exclusiva ou predominate em matas ciliares ou de várzeas, ocorrendo em solos permanentemente muito úmidos, encharcados ou brejosos, sujeitos a imundações periódicas, sendo pouco freqüentes nas matas firmes. Espécie como espeto (Casearia silvestris) inverteu a situação, apresentando valores médios de densidade/ha de 100 plantas no estrato A e 40 plantas no estrato B, enquanto que o macoqueiro (Aspidosperma sp) apresentou 200 plantas no estrato A e 20 plantas no estrato B. Esse fato pode ser atribuído à proximidade dos estratos e as características fitossociologicas da mata.

Algumas espécies apresentaram maior crescimento em altura no estrato B como por exemplo o Ingá (Inga uruguensis) com $0,23 \mathrm{~m}$ no estrato A e $1,11 \mathrm{~m}$ no estrato $\mathrm{B}$; o pessegueiro (Prunus sellowii) apresentou $0,35 \mathrm{~m}$ no esrato A e $2,12 \mathrm{~m}$ no estrato B. A pereira (Platycyamus regnelli) apresentou maior crescimento em altura no estrato A com 1,07 $\mathrm{m}$ e 0,63 metros no estrato B. No estrato com maior densidade, encontrou-se também maior valor médio em altura, esse fato pode ser atribuído à competição entre as espécies. Muitos fatores podem contribuir para que tal situação ocorra, como distância da árvore matriz, presença de agentes dispersores das sementes, tipo de agente dispersor, entre outros.

\section{CONCLUSÃO}

Nas condições em que foram realizadas neste trabalho, pode-se concluir que:

$\mathrm{Na}$ amostra mais próxima da margem do lago, encontraram-se 34 espécies com densidade de 3770 plantas /ha, enquanto que no estrato mais distante do lago, encontraram-se 29 espécies com 4510 plantas/ha.

$\mathrm{Na}$ amostra mais distante do lago, as plantas apresentaram maior altura média com 1,43 $\mathrm{m}$, enquanto que no estrato próximo ao lago, obteve-se uma altura média de 1,15 $\mathrm{m}$. Com relação ao diâmetro da base, verificou-se maior crescimento no estrato mais distante do lago com valor médio de $1,25 \mathrm{~cm}$, enquanto que no estrato próximo ao lago, obteve-se $0,97 \mathrm{~cm}$.

As espécies angico, canela amarela e jacarandá entre outras, só ocorreram no estrato próximo ao lago, enquanto que outras espécies, como cedro e açoita cavalo, apareceram apenas no estrato mais distante do lago. Espécies como canela sassafraz, canjerana entre outras, foram encontradas em 
ambas as amostras.

\section{REFERÊNCIAS BIBLIOGRÁFICAS}

ALVARENGA, R.M. Mata Atlântica. Revista Silvicultura, São Paulo, n.74, p. 26-30, 1998.

BARBOSA, L.M. Ecological significance of Gallery Forests, including biodiversity. In: INTERNATIONAL SIMPOSIUM ON ASSESMENT AND MONITORING OF FOREST IN TROPICAL DRY REGIONS WITH SPECIAL REFERENCED TO GALLERY FORESTS, 1996, Brasília. Proceedings... Brasilia: UNB, 1997. p.158-181.

CAMPOS, J.C.; LANDGRAF, P.R.C. Análise da cobertura florestal das bacia hidrográficas dos rios Cabo Verde e Machado no Sul de Minas. In: CONGRESSO FLORESTAL BRASILEIRO, 6., 1990, Campos do Jordão. Anais... São Paulo: SBS,1990. p111-117.

FINGER, Z. Estudos sobre a identificação dendrológica de regeneração natural de algumas espécies na microregião de Viçosa., Minas Gerais. Viçosa: VFV, 1977. 92p. Dissertação (Mestrado) Universidade Federal de Viçosa, 1977.

GANDOLFI, S. Estudo floristico e fitossociológico de uma floresta residual na área Aeroporto Internacional de São Paulo, município de Guarulhos, SP. Campinas: UNICAMP, 1991. 232p. Dissertação (Mestrado em Biologia Vegetal) - Universidade de Campinas, 1991.

GOLFARI, L. Zoneamento Ecológico do Estado de Minas Gerais para Reflorestamento. Belo Horizonte: Centro de Pesquisa Florestal da Região do Cerrado, 1975.

INOUE, M.T. Regeneração natural: seus problemas e perspectivas para as florestas brasileiras. Curitiba: FUPEF, 1979. 22p. ( Serie Técnica, 1)

LORENZI, H. Árvores brasileiras: manual de identificação e cultivo de plantas arbóreas do Brasil. 2.ed. Nova Odessa: Plantarum, 1998. 352p.

OLIVEIRA FILHO, A. T.; VILELA, E. A.; CARVALHO, D.A. et al. Estudos florísticos e fitossociológicos em remanescentes de matas ciliares do Alto e Médio Rio Grande. Belo Horizonte: CEMIG/UFLA/FAEPE, 1995. 27p.

PINHEIRO, A.A.L., RAMALHO, R.S., VIDAL, M.R.R. Estudo dendrológico com vista regeneração natural de Meliaceae na microregião de Viçosa, MG .Revista Árvore, v.13, n. 1, 51-65p., 1989.

RAMOS, A.E.; VALENTE, I. B. V.; NOBREGA, M. G. Estudo florístico da mata de galeria do córrego Cabeça de Veado, Estação Ecológica do Jardim Botânico de Brasília, DF. In: SIMPÓSIO SOBRE MATA CILIARES, 2., 1995, Ribeirão Preto. Resumos... Ribeirão Preto: SBB, 1995. p.327.

SEITZ, R. A . A regeneração natural na recuperação de áreas degradadas. In: SIMPÓSIO SUL AMERICANO,1.; SIMPÓSIO NACIONAL 2.; RECUPERAÇÃO DE ÁREAS DEGRADADAS, 1, Fóz de Iguaçú. Anais... Curitiba: FUPEF, 1994. p. 103110.

SPINA, A. P.; LEITÃO FILHO, H.F. Composição florística de uma floresta higrófila no município de Campinas: dados preliminares. In: CONGRESSO NACIONAL DE BOTÂNICA, 46., 1995, Ribeirão Preto. Resumos... Ribeirão Preto: SBB, 1995. p.129. 